\title{
Cobertura plástica e densidade de plantio na qualidade das frutas de Physalis peruviana L. ${ }^{1}$
}

\author{
Pedro Henrique Abreu Moura*2, Rafael Pio ${ }^{3}$, Paula Nogueira Curi ${ }^{4}$, Luiz Carlos de Almeida Rodrigues, \\ Flávio Gabriel Bianchini ${ }^{6}$, Rayane Barcelos Bisi ${ }^{7}$
}

$10.1590 / 0034-737 \times 201663030009$

\begin{abstract}
RESUMO
Physalis peruviana L. é uma espécie pertencente à família Solanaceae, que vem sendo incorporada em plantios de pequenas frutas, com alto potencial produtivo para regiões subtropicais. Objetivou-se, com este trabalho, avaliar a qualidade das frutas de P. peruviana L., produzidas com ou sem cobertura plástica sobre o dossel das plantas e em duas densidades de plantio ( 0,5 e 1,0 m entre plantas), sendo de 3,0 $\mathrm{m}$ o espaçamento entre linhas. O delineamento experimental adotado foi em blocos ao acaso, em esquema fatorial 2 × 2, com seis blocos e dez plantas por parcela. Foram avaliadas as características qualitativas das frutas, como coloração, quantidade de antocianinas totais, vitamina C, cinzas, sólidos solúveis totais, sacarose, glicose, açúcares totais, acidez titulável, firmeza e umidade. A maior densidade de plantio e o plantio sem cobertura plástica sobre o dossel das plantas resultaram em melhor qualidade das frutas de P. peruviana L.
\end{abstract}

Palavras-chave: fisális, pequenas frutas, pós-colheita, plasticultura.

\begin{abstract}
Plastic cover and planting density on the quality of Physalis peruviana $\mathrm{L}$. fruits

Physalis peruviana $\mathrm{L}$. is a species belonging to the Solanaceae family, which has been incorporated in small fruit plantations with high yielding potential for subtropical regions. The objective of this study was to evaluate the quality of $P$. peruviana $\mathrm{L}$. fruits, produced with or without plastic cover over the plant canopy and two planting densities $(0.5$ and $1.0 \mathrm{~m}$ between plants) with $3.0 \mathrm{~m}$ between planting lines. The experimental design was randomized blocks in a factorial 2 x 2 with 6 blocks and 10 plants per plot. We evaluated the qualitative characteristics of fruits such as fresh weight, length, diameter, color, amount of total anthocyanins, vitamin $\mathrm{C}$, ash, soluble solids, sucrose, glucose, total sugars, titratable acidity, firmness and moisture. The higher planting density and planting without plastic cover over the plant canopy resulted in better quality of P. peruviana L. fruits.
\end{abstract}

Key words: Physalis; small fruits; post-harvest; plasticulture.

\footnotetext{
Submetido em 24/04/2014 e Aprovado em 12/01/2016.

${ }^{1} \mathrm{O}$ presente trabalho foi extraído da tese de doutorado do primeiro autor, sendo parte da pesquisa financiada pela FAPEMIG e pela CAPES, com a colaboração dos Departamentos de Agricultura (Setor de Fruticultura) e de Ciência dos Alimentos (Laboratório de Pós-colheita de Frutas e Hortaliças) da Universidade Federal de Lavras - UFLA

${ }^{2}$ Universidade Federal de Lavras, Departamento de Agricultura, Lavras, Minas Gerais, Brasil. pedrohamoura@yahoo.com.br

${ }^{3}$ Universidade Federal de Lavras, Departamento de Agricultura, Lavras, Minas Gerais, Brasil. rafaelpio@ hotmail.com

${ }^{4}$ Universidade Federal de Lavras, Departamento de Agricultura, Lavras, Minas Gerais, Brasil. paulanogueiracuri@ yahoo.com.br

${ }^{5}$ Universidade Federal de Lavras, Departamento de Biologia, Lavras, Minas Gerais, Brasil. rodrigues.lca@gmail.com

${ }^{6}$ Universidade Federal de Lavras, Departamento de Agricultura, Lavras, Minas Gerais, Brasil. fgbianchini@yahoo.com.br

${ }^{7}$ Universidade Federal de Lavras, Departamento de Agricultura, Lavras, Minas Gerais, Brasil. rayanebbisi@gmail.com

*Autor para correspondência: pedrohamoura@yahoo.com.br
}

Rev. Ceres, Viçosa, v. 63, n.3, p. 334-339, mai/jun, 2016 


\section{INTRODUÇÃO}

A grande difusão de informações sobre as características e propriedades nutricionais de espécies como amora-preta, framboesa, morango e mirtilo tem despertado crescente interesse por parte de produtores, comerciantes, consumidores e pesquisadores, que as denominam de "pequenas frutas" (Curi et al., 2015a; Guedes et al., 2013; Maro et al., 2014; Moura et al., 2012).

Inserido recentemente no ranking das pequenas frutas, o gênero Physalis L., pertencente à família Solanaceae, possui a espécie Physalis peruviana L., que também se destaca por produzir frutas de grande valor nutricional e econômico (Gonçalves et al., 2012; Muniz et al., 2011; Rufato et al., 2012; Curi et al., 2015b).

Seu cultivo é uma excelente alternativa para os mercados nacional e internacional, por suas propriedades nutracêuticas (Rodrigues et al., 2013). Puente et al. (2011) e Ramadan (2011) relataram que as fisális apresentam teores consideráveis de vitamina $\mathrm{A}, \mathrm{B}$ e $\mathrm{C}$, além de conterem micronutrientes, como Ferro e Zinco, e macrominerais, como o Fósforo, e pequenas quantidades de Cálcio. Segundo Severo et al. (2010), essas frutas também apresentam significativos teores de compostos fenólicos e carotenoides.

Souza et al. (2014) avaliaram a composição nutricional de amora-preta, framboesa vermelha, morango, cereja doce e mirtilo, produzidos nas áreas subtropicais do Brasil, e encontraram, por exemplo, altos níveis de ácido ascórbico, também denominado Vitamina C. De acordo com Maro et al. (2012, 2013), é possível que a P. peruviana L. apresente as características nutricionais de suas frutas similares às características nutricionais dessas pequenas frutas, sendo, em alguns casos, até superiores.

Tendo em vista as exigências cada vez maiores dos consumidores em relação à qualidade, principalmente relacionada com o menor uso de agroquímicos, a utilização de cobertura plástica sobre o dossel das plantas é medida que pode favorecer a qualidade das frutas e ainda reduzir a utilização de produtos químicos no pomar, já que plantas dispostas sob cobertura recebem pouco ou quase nenhuma precipitação sob os frutos e folhas, diminuindo problemas com doenças fúngicas. De acordo com Comiran et al. (2012), esta medida já é consagrada em vinhedos, para conter os efeitos do excesso de precipitação sobre a copa da planta, especialmente durante a maturação das frutas, e com isso manter a qualidade das uvas. Curi et al. (2014) também verificaram que framboeseiras dispostas sob cobertura plástica produzem frutos de maior qualidade e com menor incidência de ferrugem, principal doença em framboesas. Diante disso, acreditase que a utilização da plasticultura, por meio de cobertura plástica sobre o dossel das plantas, possa influenciar nesses fatores, mantendo a qualidade das frutas de $P$. peruviana $\mathrm{L}$.

Outro fator importante a ser considerado é o maior aproveitamento da área de exploração, por meio do adensamento de plantio. Sendo assim, estudos são necessários para verificar se esse adensamento influenciará na qualidade das frutas.

É visto que estes dois fatores, tanto a cobertura plástica quanto o adensamento das plantas, são práticas empregadas visando a aspectos produtivos da cultura, que buscam obter maiores rendimentos por meio do melhor aproveitamento da área de plantio, da redução de aplicação de produtos químicos, como fungicidas, e também da redução de perda de frutos e do aumento da qualidade pela melhoria dos aspectos fitossanitários. Portanto, o estudo de aspectos químicos de qualidade das frutas torna-se necessário para validar estes fatores quanto à melhor aceitação das frutas pelo mercado consumidor, o qual evidencia uma busca crescente por alimentos com atividades funcionais.

Nesse sentido, objetivou-se, com este trabalho, avaliar a qualidade das frutas de $P$. peruviana $\mathrm{L}$., de acordo com a densidade de plantio e com presença ou ausência de cobertura plástica sobre o dossel das plantas.

\section{MATERIAL E MÉTODOS}

O trabalho foi conduzido de maio de 2012 a março de 2013, no município de Lavras, MG, nas dependências do Setor de Fruticultura da Universidade Federal de Lavras (UFLA). O município está situado a $21^{\circ} 14^{\prime} 06^{\prime \prime} \mathrm{S}$ e a $45^{\circ} 00^{\prime} 00^{\prime \prime} \mathrm{O}$, em altitude média de 918 metros. O clima da região é do tipo Cwb (clima mesotérmico ou tropical de altitude), com inverno seco e verão chuvoso (Alvares $e t$ al., 2013).

As mudas da Physalis peruviana L. foram produzidas por sementes, que foram semeadas em substrato à base de casca de pinus, ocorrendo sua emergência entre 20 e 30 dias após este procedimento. As mudas foram levadas ao campo entre 45 e 60 dias após a emergência, quando atingiram $15 \mathrm{~cm}$ de altura.

O plantio em campo foi realizado em maio de 2012. Adotaram-se duas densidades de plantio: $3,0 \mathrm{~m}$ x 1,0 m (densidade de 3.333 plantas por hectare) e $3,0 \mathrm{~m}$ x 0,5 m (densidade de 6.667 plantas por hectare). No momento do plantio, foram aplicados $200 \mathrm{~g}$ de calcário, $100 \mathrm{~g}$ de superfosfato simples e três litros de composto, por metro linear de sulco (Muniz et al., 2011). O composto aplicado originou-se da mistura de restos vegetais e de esterco de curral, preparada na própria universidade.

As plantas foram conduzidas em palanques, constituídos por dois mourões de eucalipto com altura de 1,6 m, espaçados de $90 \mathrm{~cm}$, com arames lisos paralelos a 1,5 m do 
solo. A condução das plantas e os tratos culturais foram realizados segundo as recomendações de Muniz et al. (2011). Todas as plantas foram irrigadas por gotejadores, colocados a cada $30 \mathrm{~cm}$, com vazão de 1,7 litros por hora em cada gotejador.

$\mathrm{O}$ delineamento adotado foi em blocos ao acaso, em esquema fatorial 2 × 2 (densidade de plantio x uso de cobertura), com seis repetições de dez plantas por parcela.

No fator uso de cobertura foi utilizado sobre as plantas o plástico leitoso PEBD, de 150 ìm de espessura, em um dos níveis. Essa cobertura foi instalada sobre fios metálicos e arcos de ferro galvanizado com 1,4 m de largura, dispostos sobre o dossel das plantas a cada $2,5 \mathrm{~m}$, os quais, por sua vez, foram fixados sobre a estrutura de mourões de eucalipto. O ponto mais alto da cobertura plástica ficou a $40 \mathrm{~cm}$ da extremidade das hastes das plantas, caracterizando um túnel baixo, adaptado à altura da cultura.

As análises foram realizadas durante o período de dezembro de 2012 e janeiro de 2013. Após a colheita, as mudas de fisális foram levadas, em bandejas de polietileno tereftalato transparente, acondicionadas em caixas de isopor com gelo, para o Laboratório de Pós-Colheita de Frutas e Hortaliças do Departamento de Ciências dos Alimentos da UFLA, onde se procedeu à seleção quanto à uniformidade de cor e ausência de injúrias mecânicas ou fisiológicas, resultando em uma amostra de 20 frutas de cada parcela. No Laboratório, as frutas foram submetidas às seguintes análises:

- Coloração: determinada em dois pontos distintos da fruta, utilizando-se o colorímetro Minolta CR-400, com a determinação no modo CIE L* a* b*. A coordenada L* refere-se ao nível de luminosidade, representando quão clara ou escura é a amostra, com valores variando de 0 (totalmente preta) a 100 (totalmente branca). Já a coordenada a* pode assumir valores de $-80 \mathrm{a}+100$, em que os extremos correspondem ao verde e ao vermelho, respectivamente. Por fim, a coordenada b*, com a intensidade de azul ao amarelo, pode variar de -50 (totalmente azul) a +70 (totalmente amarelo). As medidas foram obtidas em dois pontos diametralmente opostos, da zona equatorial da fruta e a coloração, expressa pela luminosidade $\left(\mathrm{L}^{*}\right)$, que determina o brilho, pela cromaticidade (croma), que determina a intensidade da cor, e pelo ângulo hue ( ${ }^{\circ}$ hue), que determina a tonalidade, conforme a metodologia descrita por McGuire (1992).

- Antocianinas totais: o conteúdo de antocianinas totais foi determinado pelo método da diferença de $\mathrm{pH}$, em que se dissolve em dois sistemas tampão: cloreto de potássio $\mathrm{pH} 1,0(0,025 \mathrm{M})$ e acetato de sódio $\mathrm{pH} 4,5$ $(0,4 \mathrm{M})$. Foram adicionados $2,5 \mathrm{~mL}$ da correspondente solução tampão, $\mathrm{pH}=1,0, \mathrm{a} 1,5 \mathrm{~mL}$ do extrato das fisális, e 2,5 mL da solução tampão, $\mathrm{pH}=4,5$, a 1,5 mL do extrato das fisális, sendo as leituras das absorbâncias realizadas a 510 e $700 \mathrm{~nm}$. A absorbância foi calculada a partir da equação:

$A=\left(A_{510 \mathrm{~nm}}-A_{700 \mathrm{~nm}}\right) p H_{1,0}-\left(A_{500 \mathrm{~nm}}-A_{700 \mathrm{~nm}}\right) p H_{4,5}$

A concentração de pigmentos no extrato foi calculada e representada em cianidina-3-glicosídeo, pela equação:

Antocianinas $\left(m g . l^{-1}\right)=(A \times P M \times F D \times 1000) /([x 1)$,

em que $\mathrm{A}=$ absorbância, $\mathrm{PM}=$ peso molecular, $\mathrm{FD}=$ fator de diluição $\varepsilon=$ absorvidade molar.

- Vitamina C: o teor de ácido ascórbico foi determinado pelo método colorimétrico, utilizando-se 2,4 dinitrofenilhidrazina. A leitura foi realizada em espectrofotômetro Beckman 640 B, com sistema computadorizado, e os resultados foram expressos em mg de ácido ascórbico por $100 \mathrm{~g}$ de polpa.

- Cinzas: correspondem ao resíduo obtido por incineração em temperaturas de 550-570 ÚC (AOAC, 2005).

- Sólidos solúveis totais: determinados por refratometria, conforme as normas da Association of Official Analytical Chemists - AOAC (2005), utilizando-se refratômetro digital, homogeneizando-se as amostras e transferindo-se uma ou duas gotas do material para o prisma do refratômetro. Os resultados foram expressos em ${ }^{\circ}$ Brix.

- Açúcares totais, glicose e sacarose: as extrações foram feitas pelo método de Lane-Enyon, citado pela AOAC (2005), e os açúcares totais e os redutores (glicose) foram determinados segundo a técnica de Somogy, adaptada por Nelson (1944). A leitura foi realizada em espectrofotômetro Beckman 640 B, com sistema computadorizado, e os resultados foram expressos em percentagem (g por $100 \mathrm{~g}$ de polpa). Para a determinação dos açúcares não redutores (sacarose), foi utilizada a seguinte equação:

Açúcares não redutores $=$ (açúcares totais - açúcares redutores) $\times 0,95$

- Acidez total titulável: foram retiradas amostras das frutas e procedeu-se à trituração em politron, com cinco gramas de amostra transferidas para erlenmeyers, completando-se o volume para $50 \mathrm{~mL}$ com água destilada, a que se adicionaram três gotas de indicador fenolftaleína 1\%. Procedeu-se, então, às titulações, sob agitação, com solução de hidróxido de sódio $(\mathrm{NaOH})$ 0,01 M, padronizada com biftalato de potássio. Os resultados foram expressos em percentagem de ácido cítrico. 
- Firmeza: foi medida a força necessária para que uma sonda de $3 \mathrm{~mm}$, acoplada a um penetrômetro digital, modelo Instrutherm PTR-300, vencesse a resistência da polpa da fruta. As determinações foram realizadas em dois pontos distintos das frutas, sendo os resultados expressos em Newtons (N).

- Umidade: foi determinada utilizando-se estufa a $60 \pm 5$ ÚC com circulação de ar, até a obtenção de peso constante, segundo procedimento da AOAC (2005).

- Ratio: foi obtido pelo quociente sólidos solúveis totais/ acidez total titulável.

Ao final de todas as análises, os dados foram testados, quanto à sua normalidade, pelo teste de ShapiroWilk, e posteriormente submetidos à análise de variância, sendo as médias comparadas pelo teste $\mathrm{F}$, a $5 \%$ de probabilidade.

\section{RESULTADOS E DISCUSSÃO}

Observou-se efeito significativo da interação densidade de plantio $\mathrm{x}$ uso de cobertura para tonalidade dos frutos, teores de vitamina $\mathrm{C}$ e antocininas (Tabela 2). Para o fator uso de cobertura apenas houve efeito significativo nas variáveis cinzas, sólidos solúveis e acidez titulável, em ambas as fontes de variação (Tabela 1). O ratio, o brilho dos frutos e o teor de sacarose foram afetados apenas pelo fator espaçamento (Tabela 1). As demais variáveis não apresentaram efeito significativo na interação e em cada fator isolado.

Para a coloração das fisális, a adoção de cobertura plástica sobre o dossel das plantas não afetou o brilho e a intensidade da cor, quando comparados aos das frutas produzidas em plantas descobertas. Já o espaçamento mais adensado $(3,0 \times 0,5 \mathrm{~m})$ proporcionou maior brilho $\left(\mathrm{L}^{*}\right)$ às frutas, sendo contrária a resposta para intensidade neste mesmo fator (Tabela 1).
As fisális oriundas do plantio menos adensado registraram maiores percentagens de cinzas, teor de sólidos solúveis e acidez titulável.

O valor do teor de sólidos solúveis obtido foi inferior ao encontrado por Silva et al. (2013), quando se produziram fisális, no sul de Minas Gerais, em espaçamento de $3,0 \times 1,5 \mathrm{~m}$. Esse autores relataram média superior a 14 ${ }^{\circ}$ Brix. Por outro lado, Muniz et al. (2011) obtiveram valores menores $\left(9,23^{\circ} \mathrm{Brix}\right)$ com fisális produzidas no planalto catarinense, utilizando o mesmo espaçamento e também o sistema de condução em " $X$ ".

Supõe-se que essas diferenças estejam relacionadas com as condições climáticas locais, pois, segundo Maro et al. (2013), regiões mais quentes propiciam a produção de frutas com teores mais elevados de sólidos solúveis.

Entretanto, apesar de os teores de sólidos solúveis e de a acidez titulável terem sido inferiores no espaçamento mais adensado (3,0 x 0,5 m), o ratio apresentou maior média quando a fisális foi colhida de plantas em sistema mais adensado. O mesmo foi obtido para o teor de sacarose (Tabela 1).

Em relação ao uso da cobertura, as maiores médias para cinzas, sólidos solúveis e acidez titulável foram obtidas com o uso do plástico sobre as plantas (Tabela 1).

Frutas oriundas de plantas sob plástico de cobertura apresentaram média de sólidos solúveis de $11,8^{\circ} \mathrm{Brix}$, o que representa $2,7^{\circ}$ Brix a mais, quando se compara com os resultados obtidos por Lima et al. (2012). Esses resultados, referentes ao efeito positivo da cobertura plástica no aumento de sólidos solúveis em frutas, também foram obtidos por Yamamoto et al. (2011), analisando o teor de sólidos solúveis em bagas de uva. Isso pode ser atribuído ao fato de as plantas dispostas sob coberturas plásticas apresentarem maior capacidade fotossintética, o que ocasionou o aumento da produção de açúcares, o que se reflete no maior teor de cinzas e de sólidos solúveis nas

Tabela 1: Luminosidade (L*), croma, sacarose, cinzas, firmeza, sólidos solúveis (SS), acidez titulável (AT) e ratio em frutas de $P$. peruviana L. cultivada com e sem cobertura plástica sobre o dossel das plantas e em dois espaçamentos de plantio. UFLA, Lavras, MG, 2013

\begin{tabular}{|c|c|c|c|c|c|}
\hline \multirow{2}{*}{ Variáveis } & \multicolumn{2}{|c|}{ Cobertura plástica } & \multicolumn{2}{|c|}{ Espaçamento } & \multirow{2}{*}{ CV\% } \\
\hline & Com & Sem & $3 \times 0,5 \mathrm{~m}$ & $3 \times 1,0 \mathrm{~m}$ & \\
\hline L* (brilho) & $60,2 \mathrm{a}$ & $61,3 \mathrm{a}$ & $61,7 \mathrm{a}$ & $59,7 \mathrm{~b}$ & 2,98 \\
\hline Croma (intensidade) & $46,8 \mathrm{a}$ & $46,3 \mathrm{a}$ & $46,2 \mathrm{a}$ & $46,8 \mathrm{a}$ & 2,66 \\
\hline Sacarose (mg.100g $\left.{ }^{-1}\right)$ & $2,6 \mathrm{a}$ & $2,2 \mathrm{a}$ & $2,9 \mathrm{a}$ & $1,8 \mathrm{~b}$ & 25,10 \\
\hline Cinzas $(\%)$ & $6,8 \mathrm{a}$ & $6,0 \mathrm{~b}$ & $6,1 \mathrm{~b}$ & $6,6 \mathrm{a}$ & 5,52 \\
\hline Firmeza $(\mathrm{N})$ & $3,1 \mathrm{a}$ & $3,3 \mathrm{a}$ & $3,3 \mathrm{a}$ & $3,1 \mathrm{a}$ & 11,05 \\
\hline Sólidos solúveis ( $\left.{ }^{\circ} \mathrm{Brix}\right)$ & $11,8 \mathrm{a}$ & $10,1 \mathrm{~b}$ & $10,5 \mathrm{~b}$ & $11,5 \mathrm{a}$ & 9,62 \\
\hline Acidez titulável (\%) & $1,2 \mathrm{a}$ & $1,0 \mathrm{~b}$ & $0,9 \mathrm{~b}$ & $1,2 \mathrm{a}$ & 18,59 \\
\hline Ratio (relação SS/AT) & $10,2 \mathrm{a}$ & $11,3 \mathrm{a}$ & $11,9 \mathrm{a}$ & $9,6 \mathrm{~b}$ & 23,17 \\
\hline
\end{tabular}

Médias seguidas da mesma letra nas linhas não diferem entre si pelo teste $\mathrm{F}$ a $5 \%$ de probabilidade. 
Tabela 2: Ângulo hue ( ${ }^{\circ}$ hue - tonalidade), vitamina C $\left(\mathrm{mg} \cdot 100 \mathrm{~g}^{-1}\right)$, antocianinas totais $\left(\mathrm{mg} \cdot 100 \mathrm{~g}^{-1}\right)$, açúcares totais $\left(\mathrm{mg} \cdot 100 \mathrm{~g}^{-1}\right)$, glicose (mg. $100 \mathrm{~g}^{-1}$ ) e umidade (\%) em frutas de P. peruviana L. cultivada com cobertura plástica (CC) e sem cobertura plástica (SC) sobre o dossel das plantas e em dois espaçamentos de plantio. UFLA, Lavras, MG, 2013

\begin{tabular}{|c|c|c|c|c|c|c|}
\hline \multirow{2}{*}{ Espaçamento } & \multicolumn{2}{|c|}{ Ângulo hue } & \multicolumn{2}{|c|}{ Vitamina $\mathrm{C}$} & \multicolumn{2}{|c|}{ Antocianinas totais } \\
\hline & $\mathrm{CC}$ & SC & $\mathrm{CC}$ & SC & $\mathrm{CC}$ & SC \\
\hline $3 \times 0,5 \mathrm{~m}$ & $77,6 \mathrm{Aa}$ & $76,2 \mathrm{Ba}$ & $40,2 \mathrm{Ab}$ & $43,6 \mathrm{Aa}$ & $11,6 \mathrm{Bb}$ & $35,0 \mathrm{Aa}$ \\
\hline $3 \times 1,0 \mathrm{~m}$ & $76,2 \mathrm{Ab}$ & $76,9 \mathrm{Aa}$ & $48,0 \mathrm{Aa}$ & $41,4 \mathrm{Ba}$ & $19,0 \mathrm{Ba}$ & $31,7 \mathrm{Ab}$ \\
\hline $\mathrm{CV} \%$ & & & & & & \\
\hline \multirow{2}{*}{ Espaçamentos } & \multicolumn{2}{|c|}{ Açúcares totais } & \multicolumn{2}{|c|}{ Glicose } & \multicolumn{2}{|c|}{ Umidade } \\
\hline & $\mathrm{CC}$ & $\mathrm{SC}$ & $\mathrm{CC}$ & SC & $\mathrm{CC}$ & SC \\
\hline $3 \times 0,5 \mathrm{~m}$ & $3,9 \mathrm{Ba}$ & $4,5 \mathrm{Aa}$ & $0,7 \mathrm{Bb}$ & $1,6 \mathrm{Aa}$ & $82,5 \mathrm{Aa}$ & $81,1 \mathrm{Bb}$ \\
\hline $3 \times 1,0 \mathrm{~m}$ & $3,9 \mathrm{Aa}$ & $3,4 \mathrm{Bb}$ & $1,7 \mathrm{Aa}$ & $1,7 \mathrm{Aa}$ & $82,3 \mathrm{Aa}$ & $82,4 \mathrm{Aa}$ \\
\hline $\mathrm{CV} \%$ & \multicolumn{2}{|c|}{8,69} & \multicolumn{2}{|c|}{25,45} & \multicolumn{2}{|c|}{0,76} \\
\hline
\end{tabular}

Médias seguidas pela mesma letra em maiúscula na linha e em minúscula, na coluna, não diferem entre si pelo teste $\mathrm{F}$ a $5 \%$ de probabilidade.

plantas sob cobertura plástica, em comparação com os das plantas descobertas (Tabela 1).

Analisando-se o ângulo hue, que representa a tonalidade da cor da fruta, foram obtidas as maiores médias quando se fez uso da cobertura plástica e do menor espaçamento (Tabela 2). Já dentro do menor espaçamento, as maiores médias desta variável foram com plantas sob cobertura plástica (Tabela 2). Esses valores encontrados para o ângulo hue foram muito semelhantes aos encontrados por Lima et al. (2013), que obtiveram em seus trabalhos as tonalidades de 77,32, no momento da colheita, e de 77,61, após a colheita.

Para vitamina C, a maior média dentro do uso da cobertura plástica foi obtida em frutas de plantas espaçadas em maior distância e, dentro dessa distância, com uso da cobertura plástica (Tabela 2). Verificaram-se ainda quantidades expressivas de vitamina $\mathrm{C}$, em faixas próximas ao encontrado em outras pequenas frutas, como a framboesa (Curi et al., 2014).

Por outro lado, os teores de antocianinas das frutas apresentaram maiores médias nas condições de menor e de maior espaçamento, sem uso da cobertura plástica (Tabela 2). Porém, analisando-se o efeito cobertura plástica, observaram-se respostas de superioridade divergentes, pois, com uso do plástico, a maior média foi observada no maior adensamento e, sem o uso do plástico, no menor (Tabela 2). Respostas semelhantes foram obtidas quanto ao teor de açúcares totais, pois, com o menor e o maior espaçamentos, as maiores médias foram obtidas sem e com cobertura plástica, respectivamente (Tabela 2). Além disso, sem o uso da cobertura, a maior média desta variável foi obtida com menor espaçamento, sendo o mesmo fato observado para o teor de glicose.

Entretanto, frutas produzidas por plantas sob cobertura plástica tiveram a maior média para glicose com o maior espaçamento de plantio.
Para umidade das frutas no menor espaçamento de plantio, a maior média verificou-se com o uso do plástico de cobertura, sobre as plantas e, dentro daquelas sem o uso de cobertura, a maior média foi obtida para plantio em maior espaçamento (Tabela 2).

\section{CONCLUSÕES}

Plantas mais adensadas produzem frutas com maiores brilho e teor de sacarose, menores teores de sólidos solúveis e de acidez e maior ratio.

A maioria das variáveis de qualidade, como vitamina $\mathrm{C}$, antocianinas totais, açúcares totais e glicose, apresentou maiores valores nas fisális oriundas de plantas mais adensadas (3,0 m x 0,5 m) e sem cobertura plástica.

\section{AGRADECIMENTOS}

Os autores agradecem à Fundação de Amparo à Pesquisa do Estado de Minas Gerais - FAPEMIG e a Coordenação de Aperfeiçoamento de Pessoal de Nível Superior - CAPES pelo apoio financeiro na execução deste trabalho.

\section{REFERÊNCIAS}

Alvares CA, Stape JL, Sentelhas PC, Gonçalves JLM \& Sparovek G (2013) Köppen's climate classification map for Brazil. Meteorologische Zeitschrift, 22:711-728.

AOAC - Association of Official Analytical Chemists (2005) Official methods of analysis of AOAC international. $18^{\text {th }}$ ed. Gaithersburg, AOAC International. p.20877-2417.

Comiran F, Bergamaschi H, Heckler BMM, Santos HP, Alba D \& Saretta E (2012) Microclima e produção de videiras 'Niágara Rosada' em cultivo orgânico sob cobertura plástica. Revista Brasileira de Fruticultura, 34:152-159.

Curi PN, Pio R, Moura PHA, Tadeu MH, Nogueira PV \& Pasqual M (2015a) Produção de amora-preta e amora-vermelha em Lavras - MG. Ciência Rural, 45:1368-1374. 
Curi PN, Pio R, Moura PHA, Sousa PHA, Bonfim GD \& Silva PAO (2015b) Produtividade e qualidade da framboeseira 'Batum' cultivada sob cobertura plástica e dois espaçamentos em região subtropical. Ciência Rural, 45:1994-2000.

Curi PN, Pio R, Moura PHA, Lima LCO \& Valle MHR (2014) Qualidade de framboesas sem cobertura ou cobertas sobre o dossel e em diferentes espaçamentos. Revista Brasileira de Fruticultura, 36:199-205.

Gonçalves ED, Zambon CR, Pio R, Silva LFO, Alvarenga AA \& Caproni CM (2012) Aspéctos técnicos do cultivo de fisális para o Sul de Minas. Belo Horizonte, EPAMIG. 6p. (Circular Técnica, 162).

Guedes MNS, Abreu CMP, Maro LAC, Pio R, Abreu JR \& Oliveira JO (2013) Chemical characterization and mineral levels in the fruits of blackberry cultivars grown in a tropical climate at an elevation. Acta Scientiarum: Agronomy, 35:191-196.

Lima CSM, Galarça SP, Betemps DL, Rufato AR \& Rufato L (2012) Avaliação física, química e fitoquímica de frutos de Physalis, ao longo do período de colheita. Revista Brasileira de Fruticultura, 34:1004-1012.

Lima CSM, Severo J, Andrade SB, Affonso LB, Rombaldi CV \& Rufato AR (2013) Qualidade pós-colheita de Physalis sob temperatura ambiente e refrigeração. Revista Ceres, 60:311-317.

Maro LAC, Pio R, Guedes MNS, Abreu CMP \& Curi PN (2013) Bioactive compounds, antioxidant activity and mineral composition of fruits of raspberry cultivars grown in subtropical areas in Brazil. Fruits, 68:209-217.

Maro LAC, Pio R, Silva TC \& Patto LS (2012) Ciclo de produção de cultivares de framboeseiras (Rubus idaeus) submetidas à poda drástica nas condições do sul de Minas Gerais. Revista Brasileira de Fruticultura, 34:435-441

Maro LAC, Pio R, Guedes MNS, Abreu CMP \& Moura PHA (2014) Environmental and genetic variation in the post-harvest quality of raspberries in subtropical areas in Brazil. Acta Scientiarum. Agronomy, 36:323-328

McGuire RG (1992) Reporting of objective color measurements. Hort Science, 27:1254-1255.

Moura PHA, Campagnolo MA, Pio R, Curi PN, Assis CN \& Silva TC (2012) Fenologia e produção de cultivares de framboeseiras em regiões subtropicais no Brasil. Pesquisa Agropecuária Brasileira, 47:1714-1721.
Muniz J, Kretzschmar AA, Rufato L, Pelizza TR, Marchi T, Duarte AE, Lima AAF \& Garanhani F (2011) Sistemas de condução para o cultivo de Physalis no planalto catarinense. Revista Brasileira de Fruticultura, 33:830-838

Nelson NA (1944) A photometric adaptation of Somogy method for the determination of glucose. Journal of Biological Chemists, $153: 375-384$.

Puente LA, Pinto-Muñoz CA, Castro ES \& Cortés M (2011) Physalis peruviana Linnaeus, the multiple properties of a highly functional fruit: a review. Food Research International, 44:17331740 .

Ramadan MF (2011) Bioactive phytochemicals, nutritional value, and functional properties of cape gooseberry (Physalis peruviana): an overview. Food Research International, 44:18301836.

Rodrigues FA, Penoni ES, Soares JDR \& Pasqual M (2013) Diferentes concentrações de sais do meio MS e BAP na multiplicação in vitro de Physalis peruviana L. Bioscience Journal, 29:7782 .

Rufato L, Muniz J, Kretzschmar AA, Rufato AR \& Gatiboni LC (2012) Aspectos técnicos da cultura da fisalis. Informe Agropecuário, 33:69-83.

Severo J, Lima CSM, Coelho MT, Rufato AR, Rombaldi CV \& Silva JA (2010) Atividade antioxidante e fitoquímicos em frutos de physalis (Physalis peruviana L.) durante o amadurecimento e o armazenamento. Revista Brasileira de Agrociência, 16:77-82.

Silva DF, Villa F, Barp FK, Rotili MCC \& Stumm DR (2013) Conservação pós-colheita de fisális e desempenho produtivo em condições edafoclimáticas de Minas Gerais. Revista Ceres, 60:826-832.

Souza VR, Pereira PAP, Silva TLT, Lima LCO, Pio R \& Queiroz F (2014) Determination of the bioactive compounds, antioxidant activity, and chemical composition of Brazilian blackberry, red raspberry, strawberry, blueberry and sweet cherry fruits. Food Chemistry, 156:362-368.

Yamamoto LY, Assis AM, Morais H, Souza FS, Miotto LCV, Sato AJ, Souza RT \& Roberto SR (2011) A Evolução da maturação da uva 'BRS Clara' sob cultivo protegido durante a safra fora de época. Bragantia, 70:825-831. 